\title{
Modeling of detonation transition through the field of mixing the reacting and inert gases
}

\author{
Evgeniy Prokhorov ${ }^{1, *}$ \\ ${ }^{1}$ Lavrent'ev Institute of Hydrodynamics SB RAS, 630090 Novosibirsk, Russia
}

\begin{abstract}
The non-stationary problem of exciting a plane shock wave by gas detonation in a tube is numerically solved. The case, when the field of mixing the reacting and inert gases filling the closed tube end has finite size, is considered. The influence of mixing field width on the intensity and damping law of excited shock waves is studied. Ignoring energy losses, the problem solution is determined by one dimensionless parameter equal to the ratio of gas mixture volume in the mixing field to the volume of reacting gas located in the tube before the detonation is initiated. By varying this parameter within the range from 0 to 2 , the maximal value for the Mach number of the shock wave in inert gas (air) is decreased by about $20 \%$. It is established that decrease pattern of the shock-wave front velocity can be approximately described by the dependence corresponding to the conclusions made from the theory of point explosion for the case of plane adiabatic gas motions.
\end{abstract}

The non-stationary problem of exciting a plane shock wave by gas detonation in a tube is solved numerically. In contrast to the previous theoretical papers, we consider the case (Fig. 1), where the field of mixing the reacting and inert gases located at the closed end of the tube has finite sizes $(\Delta l \neq 0)$.

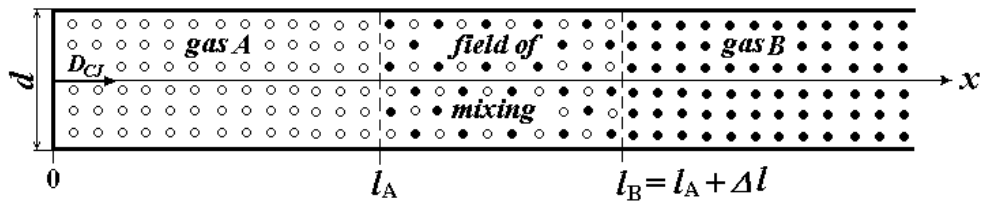

Fig. 1. The scheme of tube filling with reacting (gas A) and inert (gas B) gases before the detonation is initiated.

The mathematical statement of the problem (as in [1]) is based on the approximate model for computation of equilibrium flows of chemically reacting gases describing with high accuracy the variation of molar mass and specific internal energy in the shift of chemical equilibrium of detonation combustion products of hydrocarbons [2]. Moreover, the chemical composition of detonation products is characterized only by relative concentrations of oxygen atoms $n_{O}$, carbon atoms $n_{C}\left(n_{C} \leq n_{O}\right)$, hydrogen atoms $n_{H}$,

* Corresponding author: prokh@hydro.nsc.ru 
nitrogen atoms $n_{N}$ and any other monatomic inert substances $n_{Z}$. It is shown that such approach can be also used to describe thermodynamic properties of shock-compressed inert gas.

We performed the computational experiments simulating the process of transforming a detonation wave into a shock wave at the diffuse interface between the reacting gas (acetylene-oxygen mixture: $\mathrm{C}_{2} \mathrm{H}_{2}+2.5 \mathrm{O}_{2}$ ) and inert gas (air: $0.21 \mathrm{O}_{2}+0.78 \mathrm{~N}_{2}+0.01 \mathrm{Ar}$ ). Here it was assumed that in the field of diffusion mixing the concentrations of these gases change linearly. The initial distribution of the parameters of detonation products were given in the form of a simple wave (the Riemann wave) propagating from the rigid wall along the reacting gas with velocity of the Chapman-Jouget detonation $D_{C J}$.

We studied the influence of mixing field width $\Delta l$ on the intensity and the decay pattern of excited shock waves. To keep mass and energy balance, when varying $\Delta l$, we assumed that $l=\left(l_{A}+l_{B}\right) / 2=$ const. In this case the volume of reacting gas in the tube before the detonation is always constant, and thus it is easy to estimate its value $l=l_{A}$ at $\Delta l=0$ (Fig. 1). Then from a practical point of view the increase of $\Delta l$ can be interpreted as increasing the diffusion of interface of two contacting gases. Here the coordinate of the left boundary of mixing field $l_{A}=l-\Delta l / 2$ will shift to the closed tube end. Since within the framework of the considered statement of problem $l_{A} \geq 0$, there is upper limit for the width of mixing field equal to $\Delta l \leq 2 l$.

Ignoring energy losses (due to friction and heat removal to the tube walls), we can assume that a characteristic spatial scale of the problem is $l$. Therefore, at $\Delta l / l=$ const all calculated relationships constructed depending on dimensionless coordinate $x / l$ for different values of $l$ will coincide. Hence, the problem solution has similarity and it is defined by one dimensionless parameter $\Delta l / l$.

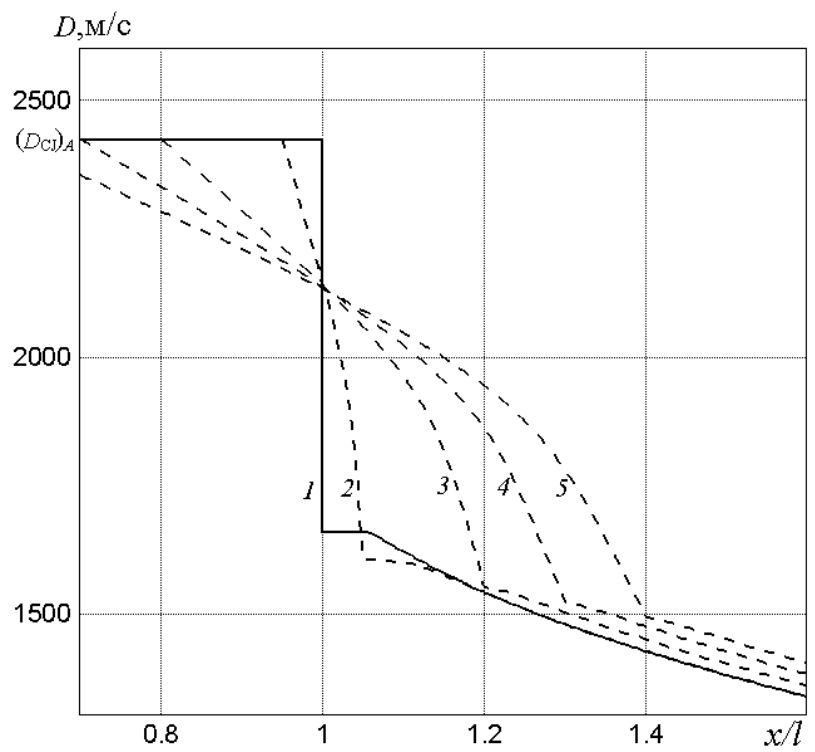

Fig. 2. Wave front velocity $D$ versus its position in the tube. Curve 1 denotes computations at $\Delta l / l=0 ; 2-\Delta l / l=0.1 ; 3-\Delta l / l=0.4 ; 4-\Delta l / l=0.6 ; 5-\Delta l / l=0.8$ 
Figure 2 demonstrates the dynamics of changing the front velocity $D$ taking place during the process of transforming a detonation wave into a shock wave at different values of mixing field width $\Delta l$. The results of computations performed for the case $\Delta l=0$, where the wave front velocity changes step-wise at the interface between the reacting and inert gases, are also shown. The rapid change of $D$ (curve 1) from the value of the Chapman-Jouget detonation velocity in gas $A$, i.e., $\left(D_{C J}\right)_{A}=2424 \mathrm{~m} / \mathrm{s}$, to the value of $\bar{D}_{0}=1658 \mathrm{~m} / \mathrm{s}$ was defined in the solution of the Riemann problem (the problem of discontinuity breakdown).

Interestingly, the proximity of slopes of all curves (see Fig. 2) takes place during the shock wave decay after passing through the mixing zone. At $x>l_{B}$, the decrease pattern of shock front velocity can be approximately described by the dependence $d(\ln D) / d(\ln x)=-1 / 2$. This result is in good agreement with the conclusions made from the theory of point explosion for the case of plane adiabatic gas motions.

Curves 2-5 also have a sharp break at $x=l_{B}\left(l_{B}=l+\Delta l / 2\right)$, where a detonation wave front transforms into a shock front. At this moment the shock front velocity is maximum. We denote it by $\bar{D}\left(\bar{D} \leq \bar{D}_{0}\right)$. Then the coordinates of the inflection point in Fig. 2 can be given as $\left(l_{B} / l, \bar{D}\right)$. The Mach number $\bar{M}=\bar{D} /\left(c_{0}\right)_{B}$ for the shock wave propagating along gas $B$ corresponds to this point.

If we draw a line passing through the inflection points of curves 2-5, it must intersect curve 1. With allowance for clarification, it is established that the intersection occurs at $x / l=1.15$ and corresponds to calculated dependence $D=D(x / l)$ at $\Delta l / l=0.3$. At $x / l>l_{B} / l=1.15$, the curve corresponding to this dependence almost (accurate to $1 \%$ ) coincides with curve 1 . The above statement is also valid for other calculated curves $D=D(x / l)$ at smaller values of $\Delta l / l$; although for them the inflection point $\left(l_{B} / l, \bar{D}\right)$ will be located below curve 1 . Here $\bar{D}$ differs slightly from $\bar{D}_{0}$. For example, for curve 2 this difference is only $3 \%$. It is obvious that curve 2 has a short horizontal segment, as curve 1 , which points to the propagation of wave with constant velocity.

This yields a practically significant conclusion that based only on measurements of shock wave front velocity in the tube part at $x / l>1.15$ in the experiment, it is impossible to answer the question whether the interface between reacting and inert gases was diffused or not up to values of $\Delta l / l=0.3$ (which may be called critical) before the detonation was initiated.

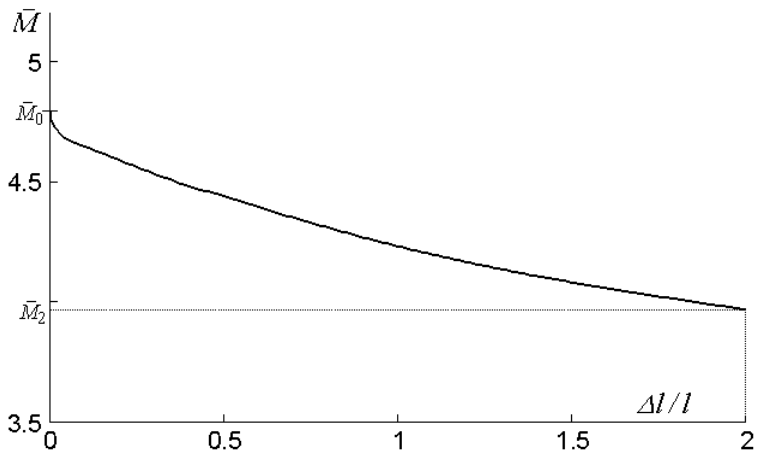

Fig. 3. Maximal Mach number of shock wave $\bar{M}$ excited by detonation in inert gas versus dimensionless parameter $\Delta l / l$. 
The dependence shown in Fig. 3 allows estimating the influence of dimensionless mixing zone width $\Delta l / l$, which defines the problem solution, on $\bar{M}$. Thus, the maximal Mach number $\bar{M}$ for a shock wave in gas $B$ has a restriction: $\bar{M}_{2}<\bar{M}<\bar{M}_{0}$, where $\bar{M}_{0}=4.791$ is found by solving the problem of discontinuity breakdown (the Riemann problem) at $\Delta l / l=0$ and $\bar{M}_{2}=3.973$ is defined at $\Delta l / l=2$. It follows that when varying parameter $\Delta l / l$, the intensity of shock wave changes slightly $\left(\bar{M}_{0} / \bar{M}_{2}=1.206\right)$. Knowing $\bar{M}$ it is easy to calculate gas-dynamic parameters of the shock front, such as density, pressure, temperature, etc., by using known formulae.

Thus, within the framework of the approach simulating the propagation of gas detonation in the medium with variable chemical composition formulated in [1], we conducted numerical solution of the problem of shock-wave excitation in refraction of the Chapman-Jouget detonation wave at diffuse interface between reacting (stoichiometric acetylene-oxygen mixture) and inert (air) gases located at the closed end of the tube. We studied the process of transformation of a detonation wave into a shock wave in the field of mixing the contacting gases, as well as analyzed the effect of mixing field width on the intensity and the decay pattern of excited shock waves.

\section{References}

1. E.S. Prokhorov, J. Eng. Phys. Thermophys. 90, 151 (2017)

2. E.S. Prokhorov, Combust. Explos. Shock Waves 32, 306 (1996) 